\title{
Two Iterative Methods for Solving Linear Interval Systems
}

\author{
Esmaeil Siahlooei ${ }^{1}{ }^{1}$ and Seyed Abolfazl Shahzadeh Fazeli $\mathbb{D I}^{1,2}$ \\ ${ }^{1}$ Department of Computer Science, Yazd University, Yazd, Iran \\ ${ }^{2}$ Parallel Processing Laboratory, Yazd University, Yazd, Iran \\ Correspondence should be addressed to Seyed Abolfazl Shahzadeh Fazeli; fazeli@yazd.ac.ir
}

Received 28 May 2018; Revised 28 August 2018; Accepted 24 September 2018; Published 8 October 2018

Academic Editor: Shyi-Ming Chen

Copyright ( 2018 Esmaeil Siahlooei and Seyed Abolfazl Shahzadeh Fazeli. This is an open access article distributed under the Creative Commons Attribution License, which permits unrestricted use, distribution, and reproduction in any medium, provided the original work is properly cited.

Conjugate gradient is an iterative method that solves a linear system $A x=b$, where $A$ is a positive definite matrix. We present this new iterative method for solving linear interval systems $\widetilde{A} \widetilde{x}=\widetilde{b}$, where $\widetilde{A}$ is a diagonally dominant interval matrix, as defined in this paper. Our method is based on conjugate gradient algorithm in the context view of interval numbers. Numerical experiments show that the new interval modified conjugate gradient method minimizes the norm of the difference of $\widetilde{A} \widetilde{x}$ and $\widetilde{b}$ at every step while the norm is sufficiently small. In addition, we present another iterative method that solves $\widetilde{A} \widetilde{x}=\widetilde{b}$, where $\widetilde{A}$ is a diagonally dominant interval matrix. This method, using the idea of steepest descent, finds exact solution $\tilde{x}$ for linear interval systems, where $\widetilde{A} \widetilde{x}=\widetilde{b}$; we present a proof that indicates that this iterative method is convergent. Also, our numerical experiments illustrate the efficiency of the proposed methods.

\section{Introduction}

Solving system of linear equations is a well-known problem in linear algebra. Many practical problems are modeled as system of linear equations. These problems have been studied by many scientists and several methods have been proposed to solve them $[1,2]$. But, in everyday life, measuring instruments just estimate values, and usually measured values are not accurate. Sometimes for more accuracy, intervals are used to represent the actual values. For example, the length of a metal rod is estimated between 24.221 and 24.222 centimeters, or temperature measured by a thermometer is between 27.6 and 27.7 Celsius.

There are many types of uncertainty and there are many different mathematical systems that calculate uncertainties [3], e.g., rough sets theory [4], fuzzy numbers [5], probability theory [6], interval valued numbers [7], and dual fuzzy numbers [8]. In this paper, uncertainty is considered as interval numbers. Some articles used this model to solve their problems $[9,10]$.

Using interval numbers in algebra was initially developed in the mid-1960s. In 1966, Moore presented his book on interval analysis [11]. Then Hansen offered a solution on interval linear algebraic equations [12]. Then many authors published their methods for solving linear interval systems, such as Neumaier, Abolmasoumi and Alavi, Nirmala, and Ganesan [13-15]. Nowadays, interval analysis methods have been applied to engineering problems, such as dynamic response analysis $[16,17]$, geotechnical structures [18], and control systems [19].

In this paper, we introduce two new iterative methods for solving a linear interval system of equations that is a linear system involving uncertain coefficients appearing as interval numbers. The solution of this system is an interval vector.

The present paper is organized as follows. The basic definitions related to interval numbers and linear interval systems are discussed in Section 3. In Section 3.1, we introduce two additional interval operations. These are inverse operations of "-" and "+." Section 3.2 recalls some properties of interval numbers and interval arithmetic. Also, it introduces some new properties on introduced operations. Next, we review conjugate gradient method and then present a new method for solving linear interval systems based on conjugate gradient method. Another new iterative method using steepest descent idea is proposed in Section 3.4. Section 4 shows the experimental results of the proposed 
methods and discusses the accuracy and efficiency of the new methods.

\section{Interval Arithmetic}

Interval numbers and arithmetic are explained in $[7,11,13$, 20]. We review main definition here. Given $a, b \in \mathbb{R}$, where $a \leq b$, the real bounded set

$$
\tilde{x}=\{x \mid a \leq x \leq b\}=[a, b]
$$

is called a proper interval. The set of all proper intervals on $\mathbb{R}$ is denoted by $\square \mathbb{R}$. In this paper, all elements in $\mathbb{R}$ are shown with a hat, i.e., $\tilde{x}$.

The infimum and supremum of $\tilde{x}=[a, b]$ are $\underline{x}=a$ and $\bar{x}=b$, respectively.

The magnitude is defined to be the maximum value of $|x|$ for all $x \in \tilde{x}$. Thus

$$
\operatorname{Mag}(\tilde{x})=\max \{A b s(x) \mid x \in \widetilde{x}\}=\max (|a|,|b|) .
$$

The width and midpoint of $\widetilde{x}$ are denoted by $w(\widetilde{x})$ and $m(\widetilde{x})$, respectively, and defined as

$$
\begin{aligned}
& w(\tilde{x})=\bar{x}-\underline{x} \\
& m(\tilde{x})=(\underline{x}+\bar{x}) / 2
\end{aligned}
$$

An interval number $\tilde{x}$ is defined as a subset of interval number $\tilde{y}$ when $\underline{x} \geq y$ and $\bar{x} \leq \bar{y}$ and denoted by $\tilde{x} \subseteq \tilde{y}$. Equality held when $\tilde{x}=\tilde{y}$.

Each real number $a \in \mathbb{R}$ can be viewed as a special interval number $\widetilde{a}=[a, a]$. The interval $[a, a]$ simply can be denoted by $a$ without confusion.

For each binary operation $*$ which is defined on $\mathbb{R}$, a binary operation $\widetilde{*}$ is defined on $\mathbb{R}$ as

$$
\tilde{x} \tilde{y} \tilde{y}=\{x * y \mid x \in \tilde{x}, y \in \tilde{y}\}
$$

for $\tilde{x}, \tilde{y} \in \mathbb{R}$. Note that we will use operator $*$ for both interval and real numbers when there is no confusion.

Therefore, basic interval arithmetic is defined in the following. Let $\tilde{x}, \tilde{y} \in \mathbb{R}$; then

$$
\begin{aligned}
& \tilde{x}+\tilde{y}=[\underline{x}+\underline{y}, \bar{x}+\bar{y}], \\
& \tilde{x}-\tilde{y}=[\underline{x}-\bar{y}, \bar{x}+\underline{y}], \\
& \tilde{x} \times \tilde{y} \\
& \quad=[\min (\underline{x} \underline{y}, \underline{x} \bar{y}, \bar{x} \underline{y}, \overline{x y}), \max (\underline{x} \underline{y}, \underline{x} \bar{y}, \bar{x} \underline{y}, \overline{x y})] . \\
& \tilde{x} / \tilde{y}=\tilde{x} \times(1 / \tilde{y}): \quad 0 \notin \tilde{y},
\end{aligned}
$$

where

$$
1 / \widetilde{y}=[1 / \bar{y}, 1 / \underline{y}]: 0 \notin \tilde{y}
$$

Note that there is a simple alternative way to formulate the interval multiplication.
An interval vector is a vector whose elements are interval numbers. Similarly, an interval matrix is a matrix whose elements are interval numbers.

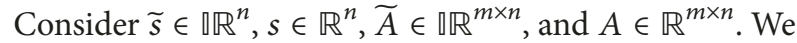
say $s \in \widetilde{s}$ if each element of vector $s$ belongs to corresponding element of $\widetilde{s}$ and $A \in \widetilde{A}$ if each element of matrix $A$ belongs to the corresponding element of $\widetilde{A}$.

Norm of interval vector $\widetilde{s}$ is defined in [21] as

$$
\operatorname{norm}(\widetilde{s})=\max _{1 \leq i \leq n}\left\{\operatorname{Mag}\left(\widetilde{s}_{i}\right)\right\}
$$

We define a square interval matrix as diagonally magnitude dominant or for simplicity diagonally dominant if

$$
\forall_{1 \leq i, j \leq n} a_{i j} \in \widetilde{a}_{i j}: A b s\left(a_{i i}\right) \geq \sum_{i \neq j} A b s\left(a_{i j}\right)
$$

Proposition 1. Interval matrix $\widetilde{A} \in \mathbb{Q} \mathbb{R}^{n \times n}$ is diagonally dominant if and only if

$$
\forall_{1 \leq i, j \leq n} \operatorname{Mig}\left(\widetilde{a}_{i i}\right) \geq \sum_{i \neq j} \operatorname{Mag}\left(\widetilde{a}_{i j}\right)
$$

Proof. Equation (13) directly follows (12); just set $a_{i i}=$ $\operatorname{Mig}\left(\widetilde{a}_{i i}\right)$ and $a_{i j}=\operatorname{Mag}\left(\widetilde{a}_{i j}\right)$ when $i \neq j$.

If (13) holds then

$$
\forall a_{i i} \in \widetilde{a}_{i i}: \operatorname{Abs}\left(a_{i i}\right) \geq \operatorname{Mig}\left(\widetilde{a}_{i i}\right)
$$

and

$$
\forall a_{i j} \in \tilde{a}_{i j}: \operatorname{Mag}\left(\tilde{a}_{i i}\right) \geq \operatorname{Abs}\left(a_{i j}\right)
$$

Finally,

$$
\begin{aligned}
\forall_{1 \leq i, j \leq n} a_{i j} & \in \widetilde{a}_{i j}: \operatorname{Abs}\left(a_{i i}\right) \geq \operatorname{Mig}\left(a_{i i}\right) \\
& \geq \sum_{i \neq j} \operatorname{Mag}\left(\tilde{a}_{i j}\right) \sum_{i \neq j} \operatorname{Abs}\left(a_{i j}\right) .
\end{aligned}
$$

General matrix form of linear interval systems can be written as follows:

$$
\widetilde{A} \widetilde{x}=\widetilde{b},
$$

where $\widetilde{A}$ is an interval matrix, $\widetilde{x}$ is an interval vector solution, and $\widetilde{b}$ is an interval vector.

To solve a linear interval system, there exist some different approaches [22-24]. Four of the well-known approaches are as follows:

(1) Find an interval vector $\tilde{x}$ such that, for all $x \in \tilde{x}, A \in$ $\widetilde{A}$ and $b \in \widetilde{b}$ exist such that $A x=b$ (united solution set).

(2) Find an interval vector $\tilde{x}$ such that, for all $x \in \tilde{x}$, for all $A \in \widetilde{A}, b \in \widetilde{b}$ exists such that $A x=b$ (tolerable solution set). Equivalently,

$$
\forall x \in \tilde{x}: \widetilde{A} x \subseteq \widetilde{b}
$$


(3) Find an interval vector $\tilde{x}$ such that, for all $x \in \tilde{x}$, for all $b \in \widetilde{b}, A \in \widetilde{A}$ exists, such that $A x=b$ (controllable solution set). Equivalently,

$$
\forall x \in \widetilde{x}: \widetilde{b} \subseteq \widetilde{A} x
$$

(4) Find an interval vector $\tilde{x}$ such that multiplication $\widetilde{A} \widetilde{x}$ is equal to $\tilde{b}$ (exact solution set).

United solutions are studied in $[13,25,26]$. In $[22,27]$ methods have been published to find tolerable solutions. Also, some methods have been developed to obtain controllable solutions [14, 28] and exact solutions [12, 14, 29-31] of interval linear systems.

Our proposed method calculates a solution for $\widetilde{A} \tilde{x}=\tilde{b}$ in the sense of the exact solution, where $\widetilde{A}$ is a diagonally dominant interval matrix.

\section{Materials and Methods}

3.1. Inverse Operations for “+” and “ $x$ ”. Suppose we are given an equation $\widetilde{a}+\tilde{x}=\widetilde{a}$, where $\widetilde{a}$ is a known interval number, and $\tilde{x}$ is an unknown interval number, how can one find $\tilde{x}$ ?

Obviously $\tilde{x}=[0,0]=0$ is the solution to this equation. However, $\widetilde{a}-\widetilde{a}=[\underline{a}-\bar{a}, \bar{a}-\underline{a}]$ which is not equal to $[0,0]$ unless $\underline{a}=\bar{a}$. If the operation "-" is inverse of the operation “+," then $\tilde{x}$ must equal $\widetilde{a}-\widetilde{a}$. But $\tilde{x}=[0,0]$ and $\widetilde{a}-\widetilde{a} \neq[0,0]$ unless $\underline{a}=\bar{a}$.
As it can be observed, the operator “-” is not inverse of the operation "+." Similarly, it can be observed that the operation "/" is not inverse of the operation " $\times$." Therefore, we propose the inverse of operations " + " and " $x$."

Before introducing inverse operations for " + " and “ $x$," we need another general type of intervals that is defined in [32, 33]. A general closed interval $[a, b]$ is identified by two real numbers $a, b \in \mathbb{R}$ and is defined as

$$
[a, b]=\{x \in \mathbb{R} \mid a \leq b \vee b \leq a\} .
$$

The general interval number space over real numbers is denoted by $\mathbb{a} \mathbb{R}^{*}$. Obviously, $\mathbb{a} \subseteq \subseteq \mathbb{R}^{*}$. All definitions (supremum, infimum, and magnitude) and operations in $\mathbb{R} \mathbb{R}^{*}$ are defined the same as $\mathbb{R}$ but computation over $\mathbb{a} \mathbb{R}^{*}$ is more complicated than $\mathbb{R}$. The arithmetic over $\square \mathbb{R}^{*}$ is well discussed in $[20,32,33]$. For example, $[1,3]+[3,1]=[4,4]$ as in (7), or $[1,2] \times[3,1]=[3,2]$ as in (8). The width of a general interval number can be negative; for example, $w([3,2])=-1$. Also, according to definition of subset, $[2,-2] \subseteq[0,0]$.

In this paper, by general interval, we mean general closed intervals.

For $\tilde{a}, \widetilde{b} \in \mathbb{R} \mathbb{R}^{*}$, the operation " $\ominus$ " is defined in $[20,33]$ as

$$
\tilde{a} \ominus \tilde{b}=[\underline{a}-\underline{b}, \bar{a}-\bar{b}]
$$

For $\tilde{a}, \tilde{b} \in \mathbb{R}$, we define the operation “ $\oslash$ " as

$$
\tilde{a} \oslash \tilde{b}= \begin{cases}{[-\infty,+\infty]} & \underline{a}=\bar{a}=0, \underline{b}=\bar{b}=0 \\ {[0,0]} & \underline{a}=\bar{a}=0,(\underline{b} \neq 0 \text { or } \bar{b} \neq 0) \\ \text { undefined } & (\exists x \neq 0, x \in \tilde{a}), \underline{b}=\bar{b}=0 \\ \text { undefined } & (0 \notin a),(0 \in b) \\ {[\underline{a} / \underline{b}, \bar{a} / \bar{b}]} & \underline{a} \geq 0, \underline{b}>0 \\ {[0, \bar{a} / \bar{b}]} & \underline{a}=0, \underline{b}=0 \\ {[\underline{a} / \bar{b}, \bar{a} / \bar{b}]} & \underline{a} \leq 0 \leq \bar{a}, \underline{b} \geq 0 \\ {[\underline{a} / \bar{b}, \bar{a} / \underline{b}]} & \bar{a} \leq 0, \underline{b}>0 \\ {[\underline{a} / \bar{b}, 0]} & \bar{a}=0, \underline{b}=0 \\ \tilde{a} \oslash(-\bar{b}) & \bar{b} \leq 0 \\ {[\underline{a} / \bar{b}, \bar{a} / \bar{b}]} & \underline{a} \geq 0, \underline{b}<0<\bar{b} \\ {[\max \{\underline{a} / \bar{b}, \bar{a} / \underline{b}\}, \min \{\bar{a} / \bar{b}, \underline{a} / \underline{b}\}]} & \underline{a} \leq 0 \leq \bar{a}, \underline{b}<0<\bar{b} \\ {[\bar{a} / \underline{b}, \underline{a} / \underline{b}]} & \bar{a} \leq 0, \underline{b}<0<\bar{b}\end{cases}
$$

Note that this definition can handle many cases where 0 belongs to $\widetilde{b}$, and just in three cases compute undefined or unbounded solution. This property lets us design algorithms with high stability and consistent when working with interval values that contain 0 .
In the following two theorems, we show that operations “ $\ominus$ " and " $\oslash$ " are inverse of operations “+” and " $x$," respectively [34].

Theorem 2. Given $\tilde{a}, \tilde{b} \in \mathbb{R}$, then the interval equation $\tilde{a}+$ $\tilde{x}^{*}=\widetilde{b}$ has a unique solution $\tilde{x}^{*}=\widetilde{b} \ominus \widetilde{a}$, where $\tilde{x}^{*} \in \mathbb{\mathbb { R } ^ { * }}$. The 
TABLE 1: Interval multiplication in detail.

\begin{tabular}{|c|c|c|c|}
\hline$\tilde{a} \tilde{x}=\widetilde{b}$ & $0 \leq \underline{a}$ & $0 \in \tilde{a}$ & $\bar{a} \leq 0$ \\
\hline & {$[\underline{a x}, \overline{a x}]$} & {$[\underline{a} \bar{x}, \overline{a x}]$} & {$[\underline{a} \bar{x}, \bar{a} \underline{x}]$} \\
\hline $0 \leq \underline{x}$ & $0 \leq \underline{b}, \bar{b}$ & $\underline{b} \leq 0 \leq \bar{b}$ & $\underline{b}, \bar{b} \leq 0$ \\
\hline $0 \in \tilde{x}$ & $\begin{array}{l}{[\bar{a} x, \overline{a x}]} \\
\underline{b} \leq 0 \leq \bar{b}\end{array}$ & $\begin{array}{c}{[\min \{\underline{a} \bar{x}, \underline{a} \bar{x}\}, \max } \\
\underline{b} \leq 0 \leq \bar{b}\end{array}$ & $\begin{array}{l}{[\underline{a} \bar{x}, \underline{a x}]} \\
\underline{b} \leq 0 \leq \bar{b}\end{array}$ \\
\hline $\bar{x} \leq 0$ & $\begin{array}{l}{[\bar{a} x, \underline{a} \bar{x}]} \\
\underline{b}, \bar{b} \leq 0\end{array}$ & $\begin{array}{l}{[\bar{a} \underline{x}, \underline{a x}]} \\
\underline{b} \leq 0 \leq \bar{b}\end{array}$ & $\begin{array}{l}{[\overline{a x}, \underline{a x}]} \\
0 \leq \underline{b}, \bar{b}\end{array}$ \\
\hline
\end{tabular}

equation $\widetilde{a}+\tilde{x}^{*}=\widetilde{b}$ has a unique solution in $\mathbb{R}$ if $\tilde{x}^{*} \in \mathbb{R}$; otherwise the equation $\widetilde{a}+\tilde{x}^{*}=\widetilde{b}$ has no solution in $\mathbb{R}$ but has exactly one in $\mathbb{R} \mathbb{R}^{*}$.

Proof. $\tilde{a}+\tilde{x}=\tilde{b} \Longleftrightarrow[\underline{a}, \bar{a}]+\left[\underline{x^{*}}, \overline{x^{*}}\right]=[\underline{b}, \bar{b}]$. That is, $\underline{b}=$ $\underline{a}+\underline{x^{*}}$, which solves $\underline{x}^{*}=\underline{b}-\underline{a}$. Similarly, $\overline{x^{*}}=\bar{b}-\bar{a}$; therefore, we have $\widetilde{x}^{*}=\tilde{b} \ominus \tilde{a}$, where $\widetilde{x}^{*}=\left[\underline{x^{*}}, \overline{x^{*}}\right]$.

Therefore if $\tilde{a}, \widetilde{b}, \tilde{x} \in \mathbb{R}$ and $\tilde{a}+\tilde{x}=\widetilde{b}$, then $\tilde{x}$ can be found with this inverse operation uniquely.

Theorem 3. Given $\tilde{a}, \tilde{b} \in \mathbb{R}$, then the interval equation $\tilde{a} \times$ $\tilde{x}^{*}=\widetilde{b}$ has a solution $\tilde{x}^{*}=\widetilde{b} \oslash \tilde{a}$, where $\tilde{x}^{*} \in \mathbb{R} \mathbb{R}^{*}$. The equation $\widetilde{a} \times \widetilde{x}^{*}=\widetilde{b}$ has a solution in $\mathbb{R}$ if $\tilde{x}^{*} \in \mathbb{R}$; otherwise the equation $\tilde{a} \times \tilde{x}^{*}=\tilde{b}$ has no solution in $\mathbb{Q} \mathbb{R}$ but has in $\mathbb{\square} \mathbb{R}^{*}$.

Remark. Solution $\tilde{x}^{*}$ is not usually unique; e.g., the interval equation $[-1,1] \times \tilde{x}^{*}=[-2,2]$ has solutions $[-1,2],[-2,0],[-2,2]$. Actually, this equation has unlimited solutions. All intervals $[u, 2]$ and $[-2, v]$ where $-2 \leq u \leq 0$ and $0 \leq v \leq 2$ can be solution of this equation.

In cases that $\tilde{x}^{*}$ is not unique, $\tilde{x}^{*}$ is considered as the longest interval as possible. In the above example, $\tilde{x}$ must be $[-2,2]$.

Proof. Suppose $\widetilde{a} \times \widetilde{x}=\widetilde{b}$. Before starting the proof, note that multiplication result, $\tilde{b}$, depends on the sign of $\widetilde{a}$ and $\tilde{x}$. Table 1 shows sign of $\tilde{b}$ related to sign of $\tilde{a}$ and $\tilde{x}$ and also shows formulas of multiplication result.

Suppose that, given $\tilde{a}, \tilde{b} \in \mathbb{R}, \tilde{a} \times \tilde{x}=\tilde{b}$, and $\bar{a} \leq 0$ and $0 \in \widetilde{b}$. Now, from Table 1 , it is observed that the following must occur: $\underline{b} \leq 0 \leq \bar{b}$ and $\tilde{b}=[\bar{a} \underline{x}, \overline{a x}]$. Then

$$
\begin{aligned}
& \tilde{a} \tilde{x}=\tilde{b} \Longleftrightarrow \\
& \tilde{b}=[\underline{a} \bar{x}, \underline{a x}] \Longleftrightarrow \\
&\left\{\begin{array}{l}
\underline{b}=\underline{a} \bar{x} \Longleftrightarrow \bar{x}=\underline{b} / \underline{x} \\
\bar{b}=\underline{a x} \Longleftrightarrow \underline{x}=\bar{b} / \underline{x}
\end{array}\right. \\
& \tilde{x}=\tilde{b} \oslash \tilde{a} .
\end{aligned}
$$

This means in this case $\tilde{x}$ is uniquely calculated by inverse operations of " $\times$." For other cases except cases with condition $0 \in \widetilde{a}$, one can provide similar proofs.

For cases with condition $0 \in \tilde{a}$, there is an ambiguity. From Table 1 , there are three cases in column $0 \in \widetilde{a}$. They have the same conditions $\underline{b} \leq 0 \leq \bar{b}$. The solution can be in forms $0 \leq x, 0 \in \tilde{x}$, and $0 \geq \bar{x}$, respectively. The solution should be in form of $0 \in \tilde{x}$, because it is the longest possible interval solution and includes both of the others. Now, find $x$ and $\bar{x}$. If $\widetilde{x_{s}}$ with condition $0 \in \widetilde{x_{s}}$ is the solution of $\widetilde{a} \times \tilde{x}=\widetilde{b}$, then $\widetilde{b}=\left[\min \left\{\bar{a} x_{s}, \underline{a} \overline{x_{s}}\right\}, \max \left\{\underline{a} \underline{x}_{s}, \overline{a x_{s}}\right\}\right]$. It follows that

$$
\begin{aligned}
\underline{b} & =\min \left\{\bar{a} x_{s}, \underline{a} \overline{x_{s}}\right\} \Longrightarrow \\
\underline{b} & \leq \bar{a} \underline{x}_{s} \\
\underline{x_{s}} & \geq \underline{b} / \bar{a},
\end{aligned}
$$

and

$$
\begin{aligned}
\bar{b} & =\max \left\{\underline{a} x_{s}, \overline{a x_{s}}\right\} \Longrightarrow \\
\bar{b} & \geq \underline{a} \underline{x_{s}} \Longrightarrow \\
\underline{x_{s}} & \geq \bar{b} / \underline{a} .
\end{aligned}
$$

Then,

$$
\underline{x_{s}} \geq \max \{\underline{b} / \bar{a}, \bar{b} / \underline{a}\} .
$$

If $\underline{x_{s}} \neq \max \{\underline{b} / \bar{a}, \bar{b} / \underline{a}\}$, then $x_{s}>\max \{\underline{b} / \bar{a}, \bar{b} / \underline{a}\}$, and $\bar{a} x_{s}>$ $\max \{\underline{b} /, \bar{a} \bar{b} / \underline{a}\} \geq \underline{b}$. This contradicts with $\bar{b}=\max \left\{\underline{a} \underline{x}_{s}, \overline{\overline{a x_{s}}}\right\}$. Hence

$$
\underline{x_{s}}=\max \{\underline{b} / \bar{a}, \bar{b} / \underline{a}\} .
$$

Similarly,

$$
\overline{x_{s}}=\min \{\bar{b} / \bar{a}, \underline{b} / \underline{a}\},
$$

and therefore, we have $\widetilde{x_{s}}=\underline{b} \oslash \underline{a}$.

In addition, special case $\widetilde{a}=[0,0]$ should be considered separately. If $\widetilde{a}=[0,0]$ and $\tilde{b} \neq[0,0]$, no $\tilde{x}$ exists such that $\tilde{a} \times \tilde{x}=\tilde{b}$. If $\widetilde{a}=\tilde{b}=[0,0]$, then $\tilde{x}$ is free and expression $\tilde{a} \times \tilde{x}=\tilde{b}$ is consistent for all $\tilde{x}$. At this point $\tilde{x}$ is considered as $[-\infty,+\infty]$.

3.2. Some Properties of the Interval Arithmetic Operations. In this section, first we recall some basic properties of interval arithmetic. For $\widetilde{a}, \widetilde{b}, \widetilde{c}, \widetilde{d} \in \mathbb{R}$ the following rules hold [35]:

$$
\begin{gathered}
\tilde{a}+\tilde{b}=\widetilde{b}+\widetilde{a} \\
\tilde{a} \times \widetilde{b}=\widetilde{b} \times \widetilde{a} \\
(\widetilde{a}+\widetilde{b})+\widetilde{c}=\widetilde{a}+(\widetilde{b}+\widetilde{c})
\end{gathered}
$$




$$
\begin{aligned}
& (\tilde{a} \times \widetilde{b}) \times \widetilde{c}=\tilde{a} \times(\widetilde{b} \times \tilde{c}) \\
& \widetilde{a} \pm \tilde{c} \subseteq \widetilde{b} \pm \widetilde{d} \quad \text { when } \tilde{a} \subseteq \widetilde{b}, \tilde{c} \subseteq \widetilde{d} \\
& w(a) \leq w(b) \quad \text { when } \tilde{a} \subseteq \tilde{b}
\end{aligned}
$$

For $\widetilde{a}, \widetilde{b}, \widetilde{c} \in \mathbb{R}$ we have

$$
\tilde{a}(\tilde{b}+\tilde{c}) \subseteq \tilde{a} \tilde{b}+\tilde{a} \widetilde{c}
$$

In relation (35), equality holds when $\underline{a}=\bar{a}$ or $\underline{b} \times \underline{c} \geq 0$.

Proposition 4. Consider $\widetilde{a}, \widetilde{b}, \widetilde{c} \in \mathbb{R}$; then

$$
\widetilde{a} \ominus(\widetilde{b}+\widetilde{c})=(\widetilde{a} \ominus \widetilde{b}) \ominus \widetilde{c}
$$

Proof. Let $\widetilde{d}=(\widetilde{a} \ominus \widetilde{b}) \ominus \widetilde{c}$; then $(\widetilde{a} \ominus \widetilde{b})=\widetilde{c}+\widetilde{d}$ and $\widetilde{a}=\widetilde{b}+\widetilde{c}+\widetilde{d}$, so

$$
\begin{aligned}
\widetilde{a} \ominus(\widetilde{b}+\widetilde{c}) & =(\widetilde{b}+\widetilde{c}+\widetilde{d}) \ominus(\widetilde{b}+\widetilde{c}) \\
& =(\widetilde{d}+(\widetilde{\mathbf{b}}+\widetilde{\mathbf{c}})) \ominus(\widetilde{\mathbf{b}}+\widetilde{\mathbf{c}})=\widetilde{d} \\
& =(\widetilde{a} \ominus \widetilde{b}) \ominus \widetilde{c},
\end{aligned}
$$

Proposition 5. For $\tilde{a}, \widetilde{b}, \widetilde{c} \in \mathbb{R}$ we have

$$
\widetilde{a} \tilde{b} \ominus \widetilde{a} \widetilde{c} \subseteq \widetilde{a}(\widetilde{b} \ominus \widetilde{c}),
$$

and equality holds when $\underline{a}=\bar{a}$ or $\underline{c} \times(\underline{b}-\underline{c}) \geq 0$.

Proof. Let $\tilde{d}=\widetilde{b} \ominus \widetilde{c}$ and then $\widetilde{b}=\widetilde{c}+\widetilde{d}$. So

$$
\begin{aligned}
& \tilde{a} \tilde{b}=\widetilde{a}(\widetilde{c}+\widetilde{d}) \subseteq \widetilde{a} \widetilde{c}+\widetilde{a} \tilde{d} \\
& \tilde{a} \tilde{b} \subseteq \widetilde{a} \widetilde{c}+\widetilde{a}(\widetilde{b} \ominus \widetilde{c}) \\
& \widetilde{a} \widetilde{b} \ominus \widetilde{a} \widetilde{c} \subseteq \widetilde{a}(\widetilde{b} \ominus \widetilde{c}) .
\end{aligned}
$$

Obviously equality holds when $\underline{a}=\bar{a}$ or $\underline{d} \times \underline{c} \geq 0$, where $\underline{d}=\underline{b}-\underline{c}$, which implies property $(36)$.

Proposition 6. For $\tilde{a}, \widetilde{b}, \widetilde{c} \in \mathbb{R}$ we have

$$
(\widetilde{b} \ominus \tilde{c}) \oslash \tilde{a} \subseteq(\widetilde{b} \oslash \tilde{a}) \ominus(\tilde{c} \oslash \tilde{a})
$$

Proof.

$$
\widetilde{a} \widetilde{b} \ominus \widetilde{a} \widetilde{c} \subseteq \widetilde{a}(\widetilde{b} \ominus \widetilde{c}),
$$

and using definition of $\oslash$ we have

$$
(\tilde{a} \widetilde{b} \ominus \tilde{a} \widetilde{c}) \oslash \widetilde{a} \subseteq \widetilde{b} \ominus \widetilde{c} .
$$

Define $\widetilde{B}=\tilde{a} \widetilde{b}$ and $\widetilde{C}=\widetilde{a} \widetilde{c}$; then $\widetilde{b}=\widetilde{B} \oslash \widetilde{a}$ and $\widetilde{c}=\widetilde{C} \oslash \widetilde{a}$. So,

$$
(\widetilde{B} \ominus \widetilde{C}) \oslash \widetilde{a} \subseteq(\widetilde{B} \oslash \widetilde{a}) \ominus(\widetilde{C} \oslash \widetilde{a})
$$

and hence (40) holds.

3.3. Modified Conjugate Gradient Method for Linear Interval Systems. Conjugate gradient method is one of the most useful techniques in solving iterative methods for solving linear system of equations, whose matrix is symmetric and positive definite. The conjugate gradient method was proposed by Hestenes and Stiefel in 1952 as an iterative method for solving linear systems with positive definite coefficient matrices [36]. The conjugate gradient algorithm for linear system of equations can be briefly described as follows.

Consider system $A x=b$ with solution $y$. Start with an initial estimate $x_{0}$ of $y$. At step $i$ use $x_{i}$ to new estimate $x_{i+1}$ of $y$ which is closer to $y$. At each step residual $r_{i}=b-A x_{i}$ is computed and used as a measure of the goodness of the estimate $x_{i}$. The algorithm is detailed below for solving $A x=$ $b$, where $\mathrm{A}$ is a symmetric, positive definite matrix.

We generalize this method for linear interval systems when the interval matrix $\widetilde{A}$ is symmetric, $\widetilde{A}_{i j}=\widetilde{A}_{j i}$ for all $1<=i, j<=n$, and $\widetilde{b}$ is an interval vector. There are articles describing and analyzing conjugate gradient method such as [37-39]. These articles illustrated which operator was used in original role, or acted in inverse operator. The new algorithm is obtained from original conjugate gradient method by modifying and replacing all operations with related interval operations; also all operators acting in the role of inverse operators are replaced by inverse of interval operators. In addition, we use interval vector norm instead of real vector norm.

In the first modification on conjugate gradient, we change some operation "-," with inverse operation " $\ominus$ " whenever needed. In Step 2 of Algorithm 1, "-" is applied for computing difference of $b$ and $A \times x_{0}$. In Step 7 operation "-" is used, but at proof of conjugate gradient method in [39], $r_{i}$ is replaced with expression $\left(r_{i+1}+\left(\alpha_{i} \times A p_{i}\right)\right)$. So, the operation " + " is summed over $r_{i+1}$ and $\alpha_{i} \times A p_{i}$. This means "-" in Step 7 is used for inverse of "+." Therefore we use the operation " $\ominus$ " instead of "-" in steps 2 and 7.

The second modification is similar to the first modification. We change operation "/" with inverse operation " $\oslash$." In steps 5 and 8 of Algorithm 1 operation "/" is used as inverse of multiplication in proof of conjugate gradient [39].

The third modification, norm $\left(\widetilde{r}_{i}\right)^{2}$, is used instead of $r_{i}{ }^{\prime} r_{i}$. In real linear algebra, expression $r_{i}^{\prime} r_{i}$ equals square of norm2 of $r_{i}$, and value of $r_{i}{ }^{\prime} r_{i}$ is related to amount of error. We replace this with norm $\left(\widetilde{r}_{i}\right)^{2}$ that has the same meaning. The interval modified conjugate gradient method is the result of these modifications and is shown in Algorithm 2.

3.4. Interval Steepest Descent Method for Linear Interval Systems. First, we define derivative on interval functions. In differential algebra, the derivative of $f(x)$ with respect to $x$ 

(1) function $\mathbf{C G}\left(A, b, x_{0}, n\right)$
$\triangleright$ Input: $A \in \mathbb{R}^{n \times n}, b \in \mathbb{R}^{n}, x_{0} \in \mathbb{R}^{n}$
$\triangleright$ Output: $x_{0} \in \mathbb{R}^{n}$
(2) $p_{0}=r_{0}=b-A x_{0}$
(3) $i=0$
(4) repeat$$
\alpha_{i}=\frac{r_{i}^{T} r_{i}}{\left(p_{i}\right)^{T}\left(A p_{i}\right)}
$$$$
\text { (6) } \quad x_{i+1}=x_{i}+\left(\alpha_{i} \times p_{i}\right)
$$$$
\text { (7) } \quad r_{i+1}=r_{i}-\left(\alpha_{i} \times A p_{i}\right)
$$$$
\text { (8) } \quad \beta_{i}=\frac{r_{i+1}^{T} r_{i+1}}{r_{i}^{T} r_{i}}
$$$$
\text { (9) } \quad p_{i+1}=r_{i+1}+\beta_{i} p_{i}
$$$$
\text { (10) } \quad i=i+1
$$
(11) until ( $r_{i}$ is sufficiently small)
(12) $x=x_{0}$

Algorithm 1: Conjugate gradient algorithm.

(1) function $\operatorname{ICG}\left(\widehat{A}, \widehat{b}, \widehat{x}_{0}, n, \theta, \delta\right)$

$\triangleright$ Input: $\widehat{A} \in \mathbb{Q} \mathbb{R}^{n \times n}, \widehat{b} \in \mathbb{\mathbb { R } ^ { n }}, \widehat{x}_{0} \in \mathbb{R}^{n}, \theta . \delta \in \mathbb{R}$

$\triangleright$ Output: $\hat{x} \in \mathbb{\mathbb { R } ^ { n }}$

(2) $\widehat{p}_{0}=\widehat{r}_{0}=\widehat{b} \ominus\left(A \times \widehat{x}_{0}\right)$

(3) $i=0$

(4) $N_{r}=\operatorname{norm}\left(\widehat{r}_{0}\right)^{2}$

(5) repeat

(6) $\widehat{u}=\widehat{A} \times \widehat{p}_{i}$

(7) $\widehat{\alpha}_{i}=N_{r} \oslash\left(\hat{p}_{i}^{T} \times \widehat{u}\right)$

(8) $\hat{x}_{i+1}=\hat{x}_{i}+\left(\widehat{\alpha}_{i} \times \widehat{p}_{i}\right)$

(9) $\widehat{r}_{i+1}=\widehat{r}_{i} \ominus\left(\widehat{\alpha}_{i} \times \widehat{p}_{i}\right)$

(10) $M_{r}=\operatorname{norm}\left(\widehat{r}_{i+1}\right)$

(11) if $M_{r}<\theta$ then

(12) break

(13) $\beta=\left(M_{r}\right)^{2} / N_{r}$

(14) $\widehat{p}_{i+1}=\widehat{r}_{i+1}+\left(\beta \times \widehat{p}_{i}\right)$

(15) $N_{r}=\left(M_{r}\right)^{2}$

(16) if $\operatorname{norm}\left(\hat{x}_{i} \ominus \widehat{x}_{i+1}\right)<\delta$ then

(17) break

(18) $i=i+1$

(19) until

return $\hat{x}_{i}$

Algorithm 2: Interval modified conjugate gradient algorithm.

measures the sensitivity to change value of $f(x)$ with respect to $x$. In mathematical terms

$$
f^{\prime}(x)=\lim _{h \rightarrow 0} \frac{f(x+h)-f(x)}{h}
$$

According to this, the derivative of an interval function $f(\tilde{x})$ with respect to $\tilde{x}$ is denoted as $f^{\prime}(\tilde{x})$ or $f^{\prime}{ }_{\bar{x}}(\tilde{x})$ or $(d / d \tilde{x}) f(\tilde{x})$ and is defined by the following formula:

$$
f^{\prime}(\tilde{x})=\lim _{\tilde{h} \longrightarrow[0,0]}((f(\tilde{x}+\widetilde{h}) \ominus f(\tilde{x})) \oslash \widetilde{h})
$$

Let $\tilde{x} \in \mathbb{\mathbb { R } ^ { n }}$ and $f(\tilde{x})=f\left(\widetilde{x}_{1}, \widetilde{x}_{2}, \ldots, \tilde{x}_{n}\right)$ be a $\square \mathbb{R}^{n} \longrightarrow \mathbb{R}$ function. The partial derivative of a multivariable interval function is a derivative with respect to one variable when all other variables are fixed. The partial derivative $f$ with respect to $\widetilde{x}_{i}$ is denoted as $f^{\prime} \tilde{x}_{i}(\tilde{x})$ and is defined as

$$
\begin{aligned}
& f^{\prime} \widetilde{x}_{i}(\tilde{x})=\lim _{\tilde{h} \longrightarrow[0,0]}\left(\left(f^{\prime} \widetilde{x}_{i}\left(\widetilde{x}_{1}, \ldots, \tilde{x}_{i}+\widetilde{h}, \ldots, \widetilde{x}_{n}\right)\right.\right. \\
& \left.\left.\quad \ominus f^{\prime}{\widetilde{x_{i}}}\left(\tilde{x}_{1}, \ldots, \tilde{x}_{i}, \ldots, \tilde{x}_{n}\right)\right) \oslash \widetilde{h}\right) .
\end{aligned}
$$

Immediately from definitions (44) and (45) and the properties discussed, one can obtain the following properties of derivative and partial derivative of interval functions:

$$
\begin{aligned}
&(\tilde{x})^{\prime}=1 \\
& f^{\prime}(\tilde{x})=0 \\
&(f \pm g)^{\prime}(\tilde{x})=f^{\prime}(\tilde{x}) \pm g^{\prime}(\tilde{x}) \\
&(\widetilde{t} \times f)^{\prime}(\tilde{x}) \subseteq \tilde{t} \times\left(f^{\prime}(\tilde{x})\right), \quad \tilde{t} \in \mathbb{R} \\
&(f \times g)^{\prime}(\tilde{x}) \subseteq\left(f^{\prime}(\tilde{x}) \times g(\tilde{x})\right)+\left(g^{\prime}(\tilde{x}) \times f(\tilde{x})\right)
\end{aligned}
$$

Now consider the linear interval system $\widetilde{A} \widetilde{x}=\widetilde{b}$ when $\widetilde{A} \epsilon$ $\square \mathbb{R}^{n \times n}$ is a symmetric interval matrix, and $\widetilde{b} \in \mathbb{Q} \mathbb{R}^{n}$ is a known interval vector. Let $\Phi(\tilde{x})$ be an interval function

$$
\Phi(\tilde{x})=\frac{1}{2} \tilde{x}^{T} \widetilde{A} \tilde{x} \ominus \tilde{x}^{T} \widetilde{b} .
$$

The gradient of $\Phi(\widetilde{x})$ with respect to $\tilde{x}$ is the vector of partial derivatives as

$$
\nabla_{\tilde{x}} \Phi(\tilde{x})=\left[\begin{array}{c}
\Phi_{\tilde{x}_{1}}^{\prime}(\tilde{x}) \\
\Phi_{\tilde{x}_{2}}^{\prime}(\tilde{x}) \\
\vdots \\
\Phi_{\tilde{x}_{n}}^{\prime}(\tilde{x})
\end{array}\right] .
$$

Let $f(\widetilde{x})=\widetilde{x}^{T} \widetilde{b}$ and $g(\widetilde{x})=\widetilde{x}^{T} \widetilde{A} \widetilde{x}$. Then

$$
f(\widetilde{x})=\sum_{i=1}^{n} \widetilde{b}_{i} \tilde{x}_{i}
$$

so, for any $k(1 \leq k \leq n)$

$$
\begin{aligned}
f_{\widetilde{x}_{k}}{ }^{\prime}(\widetilde{x}) & =\frac{d}{d \widetilde{x}_{k}} \sum_{i=1}^{n}\left(\widetilde{b}_{i} \widetilde{x}_{i}\right) \\
& \subseteq \frac{d}{d \widetilde{x}_{k}}\left(\sum_{i \neq k}\left(\widetilde{b}_{i} \widetilde{x}_{i}\right)\right)+\frac{d}{d \widetilde{x}_{k}}\left(\widetilde{b}_{k} \widetilde{x}_{k}\right)=0+\widetilde{b}_{k} \Longrightarrow \\
f_{\widetilde{x}_{k}}{ }^{\prime}(\widetilde{x}) & \subseteq \widetilde{b}_{k}
\end{aligned}
$$

And,

$$
g(\tilde{x})=\sum_{i=1}^{n} \sum_{j=1}^{n} \widetilde{x}_{i} \widetilde{A}_{i j} \widetilde{x}_{j}
$$

so, for all $k(1 \leq k \leq n)$

$$
g^{\prime} \widetilde{x}_{k}(\widetilde{x})=\frac{d}{d \tilde{x}_{k}} \sum_{i=1}^{n} \sum_{j=1}^{n} \widetilde{x}_{i} \widetilde{A}_{i j} \widetilde{x}_{j}=\frac{d}{d \widetilde{x}_{k}}\left(\sum_{i \neq k} \sum_{j \neq k} \widetilde{x}_{i} \widetilde{A}_{i j} \widetilde{x}_{j}\right.
$$




$$
\begin{aligned}
& \left.+\sum_{i \neq k} \widetilde{x}_{i} \widetilde{A}_{i k} \widetilde{x}_{k}+\sum_{j \neq k} \widetilde{x}_{k} \widetilde{A}_{k j} \widetilde{x}_{j}+\widetilde{x}_{k} \widetilde{A}_{k k} \widetilde{x}_{k}\right) \Longrightarrow \\
& g^{\prime} \widetilde{x}_{k}(\widetilde{x}) \subseteq 0+\sum_{i \neq k} \widetilde{x}_{i} \widetilde{A}_{i k}+\sum_{j \neq k} \widetilde{A}_{k j} \widetilde{x}_{j}+\left(\widetilde{A}_{k k} \widetilde{x}_{k}\right. \\
& \left.+\widetilde{x}_{k} \widetilde{A}_{k k}\right)=2 \times \sum_{i=1}^{n} \widetilde{A}_{k i} \widetilde{x}_{i} \Longrightarrow \\
& g^{\prime} \widetilde{x}_{k}(\widetilde{x}) \subseteq 2 \times \widetilde{A}_{k}^{T} \widetilde{x}
\end{aligned}
$$

Note that $\Phi(\widetilde{x})=(1 / 2) g(\widetilde{x}) \ominus f(\widetilde{x})$. Then

$$
\Phi_{\widetilde{x}_{k}}{ }^{\prime}(\tilde{x}) \subseteq \frac{1}{2} g_{\widetilde{x}_{k}}{ }^{\prime}(\widetilde{x}) \ominus{f_{\widetilde{x}_{k}}}^{\prime}(\widetilde{x}) \subseteq \widetilde{A}_{k}^{T} \widetilde{x} \ominus \widetilde{b}_{k}
$$

and then, from (52)

$$
\nabla_{\widetilde{x}} \Phi(\tilde{x})=\left[\begin{array}{c}
\Phi^{\prime}{\widetilde{x_{1}}}_{(\tilde{x})} \\
\Phi_{\widetilde{x}_{2}}^{\prime}(\tilde{x}) \\
\vdots \\
\Phi_{\widetilde{x}_{n}}^{\prime}(\tilde{x})
\end{array}\right] \subseteq\left[\begin{array}{c}
\widetilde{A}_{1}^{T} \tilde{x} \ominus \widetilde{b}_{1} \\
\widetilde{A}_{2}^{T} \tilde{x} \ominus \widetilde{b}_{2} \\
\vdots \\
\widetilde{A}_{n}^{T} \tilde{x} \ominus \widetilde{b}_{n}
\end{array}\right]=\widetilde{A} \tilde{x} \ominus \widetilde{b}
$$

Assume $\widetilde{x}_{*}$ is the solution of linear interval system $\widetilde{A} \widetilde{x}=\widetilde{b}$; then

$$
\begin{aligned}
2 \times \Phi(\widetilde{x}) & =\widetilde{x}^{T} \widetilde{A} \widetilde{x} \ominus 2 \widetilde{x}^{T} \widetilde{b} \\
& =\left(\widetilde{x}^{T} \widetilde{A} \widetilde{x} \ominus \widetilde{x}^{T} \widetilde{b}\right) \ominus \widetilde{x}^{T} \widetilde{b} \\
& \subseteq \widetilde{x}^{T}(\widetilde{A} \tilde{x} \ominus \widetilde{b}) \ominus \widetilde{x}^{T} \widetilde{b} \Longrightarrow \\
\Phi(\widetilde{x}) & \subseteq \frac{1}{2}\left(\widetilde{x}^{T}(\widetilde{A} \widetilde{x} \ominus \widetilde{b}) \ominus \widetilde{x}^{T} \widetilde{b}\right) .
\end{aligned}
$$

So,

$$
\begin{gathered}
\Phi\left(\widetilde{x}_{*}\right) \subseteq \frac{1}{2}\left([0,0] \ominus \widetilde{x}_{*}^{T} \widetilde{b}\right) \Longrightarrow \\
w\left(\Phi\left(\widetilde{x}_{*}\right)\right) \leq-\frac{1}{2} w\left(\widetilde{x}_{*}^{T} \widetilde{b}\right)=\text { constant value }
\end{gathered}
$$

Now, consider $\tilde{x} \in \mathbb{\mathbb { R } ^ { n }}$ and $d \in \mathbb{R}^{n}$, where $k^{\text {th }}$ element of $d$ is $\left(-w(\widetilde{A} \tilde{x} \ominus \widetilde{b})_{k}\right)$ and other elements of $d$ are zero $(1 \leq k \leq$ $n)$. Note that $d$ indicates the opposite of direction $w\left(\nabla_{\tilde{x}} \Phi(\tilde{x})\right)$. Then

$$
\begin{aligned}
\Phi(\tilde{x}+\widetilde{\alpha} d)= & \frac{1}{2}(\widetilde{x}+\widetilde{\alpha} d)^{T} \widetilde{A}(\widetilde{x}+\widetilde{\alpha} d) \ominus(\widetilde{x}+\widetilde{\alpha} d)^{T} \widetilde{b} \\
\subseteq & \frac{1}{2} \widetilde{\alpha}^{2} d^{T} \widetilde{A} d+\widetilde{\alpha} d^{T} \widetilde{A} d+\frac{1}{2} \widetilde{\alpha}^{2} \widetilde{x} \widetilde{A} \widetilde{x} \\
& \ominus \widetilde{\alpha} d^{T} \widetilde{b} \ominus \widetilde{x}^{T} \widetilde{b} .
\end{aligned}
$$

The derivative of $\Phi(\widetilde{x}+\widetilde{\alpha} d)$ with respect to $\widetilde{\alpha}$ is

$$
\Phi^{\prime} \widetilde{\alpha}(\widetilde{x}+\widetilde{\alpha} d) \subseteq d^{T} \widetilde{A} \widetilde{x}+\widetilde{\alpha} d^{T} \widetilde{A} d \ominus d^{T} \widetilde{b} .
$$

If $d^{T} \widetilde{A} \widetilde{x}+\widetilde{\alpha} d^{T} \widetilde{A} d \ominus d^{T} \widetilde{b}$ equals zero, then one can obtain $\widetilde{\alpha}$ as

$$
\begin{aligned}
\widetilde{\alpha} & =\left(d^{T} \widetilde{b} \ominus d^{T} \widetilde{A} \widetilde{x}\right) \oslash\left(d^{T} \widetilde{A} d\right) \\
& =\left(d_{k} b_{k} \ominus d_{k}(\widetilde{A} \widetilde{x})_{k}\right) \oslash\left(d_{k}^{2} \widetilde{a}_{k k}\right) \\
& =\left(b_{k} \ominus(\widetilde{A} \widetilde{x})_{k}\right) \oslash\left(d_{k} \widetilde{a}_{k k}\right) \Longrightarrow \\
\widetilde{\alpha} & =\left(\widetilde{r}_{k}\right) \oslash\left(d_{k} \widetilde{a}_{k k}\right)
\end{aligned}
$$

Now $\widetilde{\alpha}$ moves $\Phi(\widetilde{x})$ to $\Phi(\widetilde{x}+\widetilde{\alpha} d)$ with respect to direction $d$ when $\tilde{x}$ is fixed. So $\Phi(\widetilde{x}+\widetilde{\alpha} d) \subseteq \Phi(\widetilde{x})$ and also $w(\Phi(\widetilde{x}+\widetilde{\alpha} d)) \leq$ $w(\Phi(\tilde{x}))$.

$$
\begin{aligned}
& \Phi(\tilde{x}+\widetilde{\alpha} d) \ominus \Phi(\widetilde{x}) \\
& \subseteq \frac{1}{2}(\widetilde{\alpha} d)^{T} \widetilde{A}(\widetilde{\alpha} d)^{T}+(\widetilde{\alpha} d)^{T} \widetilde{A} \widetilde{x} \ominus(\widetilde{\alpha} d)^{T} b \\
& \subseteq \frac{1}{2}(\widetilde{\alpha} d)^{T} \widetilde{A}(\widetilde{\alpha} d)^{T}+(\widetilde{\alpha} d)^{T}(\widetilde{A} \widetilde{x} \ominus b) \\
&= \frac{1}{2}(\widetilde{\alpha} d)^{T} \widetilde{A}(\widetilde{\alpha} d)^{T} \ominus(\widetilde{\alpha} d)^{T}(\widetilde{r}) \\
&= \frac{1}{2}\left(\widetilde{\alpha} \widetilde{\alpha} d_{k}^{2} \widetilde{a}_{k k}\right) \ominus\left(\widetilde{\alpha} d_{k}\right)\left(r_{k}\right) \\
& \subseteq\left.\frac{1}{2}\left(\widetilde{r}_{k} \oslash\left(d_{k} \widetilde{a}_{k k}\right)\right) d_{k}\left(\widetilde{r}_{k} \oslash\left(d_{k} \widetilde{a}_{k k}\right)\right) d_{k} \widetilde{a}_{k k}\right) \\
& \ominus\left(\widetilde{r}_{k} \oslash\left(d_{k} \widetilde{a}_{k k}\right)\right) d_{k}\left(r_{k}\right) \\
&= \frac{1}{2}\left(\widetilde{r}_{k} \widetilde{r}_{k} \oslash\left(\widetilde{a}_{k k}\right)\right) \ominus\left(\widetilde{r}_{k} \widetilde{r}_{k} \oslash\left(\widetilde{a}_{k k}\right)\right) \\
&= {[0,0] \ominus \frac{1}{2}\left(\widetilde{r}_{k} \widetilde{r}_{k} \oslash\left(\widetilde{a}_{k k}\right)\right) \subseteq[0,0] \Longrightarrow } \\
& \Phi(\widetilde{x}+\widetilde{\alpha} d) \subseteq \Phi(\widetilde{x})
\end{aligned}
$$

In the following, we assume that $\widetilde{x}_{0}$ is a given vector as initial guess. If set $\widetilde{x}_{n+1}=\widetilde{x}_{n}+\widetilde{\alpha}_{n} d_{n}$, then $\Phi \widetilde{x}_{n+1} \subseteq \Phi\left(\widetilde{x}_{n}\right)$, where $\widetilde{\alpha}_{n}=\left(d_{n}{ }^{T} \widetilde{b} \ominus d_{n} \widetilde{A} \widetilde{x}_{n}\right) \oslash\left(d_{n}{ }^{T} \widetilde{A} d_{n}\right)$, have

$$
w\left(\Phi \widetilde{x}_{n+1}\right) \leq w\left(\Phi\left(\widetilde{x}_{n}\right)\right)
$$

and then

$$
\begin{aligned}
w\left(\Phi\left(\tilde{x}_{0}\right)\right) & \geq w\left(\Phi\left(\tilde{x}_{1}\right)\right) \geq w\left(\Phi\left(\tilde{x}_{2}\right)\right) \geq \ldots \\
& \geq w\left(\Phi\left(\tilde{x}_{*}\right)\right)
\end{aligned}
$$

The descending series $\left\{w\left(\Phi\left(\tilde{x}_{i}\right)\right)\right\}$ converges to $w\left(\Phi\left(\tilde{x}_{*}\right)\right)$. So, $\left\{\tilde{x}_{i}\right\}$ converges to $\tilde{x}_{*}$.

The steepest descent algorithm for intervals is shown in Algorithm 3.

\section{Results and Discussion}

In this section, some numerical examples show how our iterative methods can solve interval systems. Accuracy of produced solutions is measured by a kind of norm of residual vector

$$
r_{i}=\widetilde{A} \widetilde{x_{i}} \ominus \widetilde{b},
$$




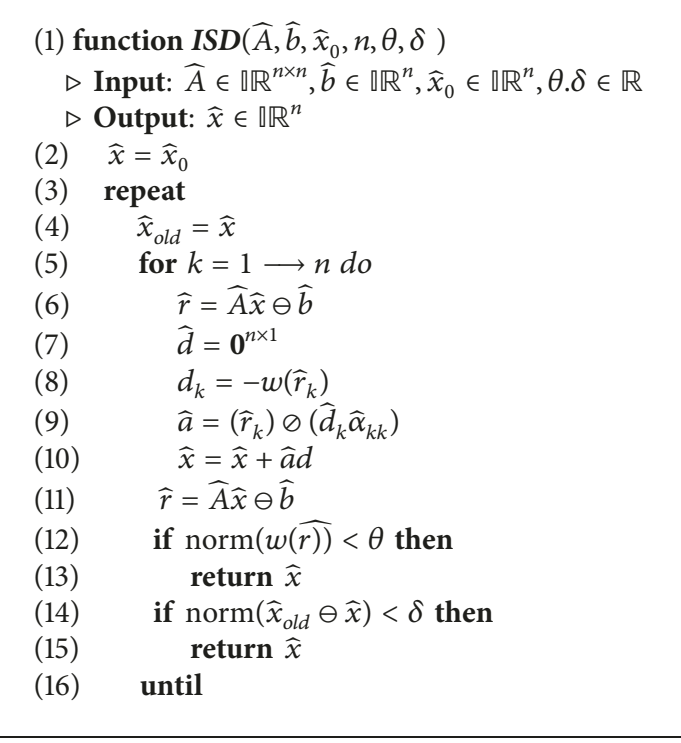

Algorithm 3: Interval steepest descent algorithm.

where $\widetilde{x_{i}}$ is the produced solution in $i$ th iteration. The norm used in this section is then maximum of magnitude of all residual vector elements.

We examine our methods with a famous system and then examine them with random matrices with several dimensions. Our results illustrate the effectiveness of our algorithms.

Example 7. Consider the linear interval system $\widetilde{A} \widetilde{x}=\widetilde{b}[22$, 40]

$$
\begin{aligned}
& \widetilde{A}=\left[\begin{array}{ccc}
{[3.74 .3]} & {[-1.5,-0.5]} & {[0,0]} \\
{[-1.5,-0.5]} & {[3.7,4.3]} & {[-1.5,0.5]} \\
{[0,0]} & {[-1.5,-0.5]} & {[3.7,4.3]}
\end{array}\right] \\
& \widetilde{b}=\left[\begin{array}{c}
{[-14,14]} \\
{[-9,9]} \\
{[-3,3]}
\end{array}\right] .
\end{aligned}
$$

Let $\tilde{x}_{0}=[0,0,0]^{T}$ be initial estimation and $\theta=10^{-3}$ be tolerance. Interval modified conjugate gradient method finds the estimation $\tilde{x}$ at iteration 13 . However, interval steepest descent finds solution $\tilde{y}$ at iteration 4

$$
\begin{gathered}
\tilde{x}=\left[\begin{array}{l}
{[-2.92653,2.92653]} \\
{[-0.94373,0.94373]} \\
{[-0.36839,0.36839]}
\end{array}\right], \\
\tilde{y}=\left[\begin{array}{l}
{[-2.92639,2.92639]} \\
{[-0.94373,0.94373]} \\
{[-0.36846,0.36846]}
\end{array}\right] .
\end{gathered}
$$

Accuracy of results is determined by the residual vectors as follows:

$$
\begin{aligned}
& \operatorname{Mag}(\tilde{b} \ominus A \tilde{x})=\left[\begin{array}{l}
0.319044 \\
0.436095 \\
0.319044
\end{array}\right] 10^{-4}, \\
& \operatorname{Mag}(\tilde{b} \ominus A \tilde{y})=\left[\begin{array}{c}
0.923864 \\
0.322278 \\
0
\end{array}\right] 10^{-3}
\end{aligned}
$$

Also when $\theta=10^{-7}$ the accuracy of the obtained results of two methods is

$$
\operatorname{Mag}(\widetilde{b} \ominus A \tilde{x})=\left[\begin{array}{l}
0.39509 \\
0.56648 \\
0.39509
\end{array}\right] 10^{-7},
$$

$$
\operatorname{Mag}(\tilde{b} \ominus A \widetilde{y})=\left[\begin{array}{c}
0.46724 \\
0.16299 \\
0
\end{array}\right] 10^{-7}
$$

when $\tilde{x}$ and $\tilde{y}$ are found at $26^{\text {th }}$ and $11^{\text {th }}$ iterations, respectively.

Tables 2 and 3 show values $\tilde{x}_{i}$ and $\tilde{y}_{i}$ with their maximum of the magnitude of the residual vector at $i^{t h}$ iteration, respectively.

The method presented in $[13,15,25,26,30]$ could not solve this system where 0 belongs to their elements to the right-hand side vector, because their methods cannot resolve divide-by-zero issues.

This system is solved in $[22,40]$, and solutions obtained by them are

$$
\begin{aligned}
& \widetilde{z}_{1}=\left[\begin{array}{c}
{[-2.3802,-1.7730]} \\
{[-2.0827,-1.8280]} \\
{[-0.8482,-0.8482]}
\end{array}\right], \\
& \widetilde{z}_{2}=\left[\begin{array}{c}
{[-1.6700,6.3800]} \\
{[-2.7700,6.4000]} \\
{[-2.400,3.400]}
\end{array}\right],
\end{aligned}
$$

respectively.

Note that

$$
\begin{aligned}
& A \widetilde{z}_{1}=\left[\begin{array}{l}
{[-9.3209,-3.4360]} \\
{[-7.6451,-1.9210]} \\
{[-2.7333,-0.0142]}
\end{array}\right], \\
& A \widetilde{z}_{2}=\left[\begin{array}{l}
{[-16.7810,31.5890]} \\
{[-26.5811,33.6251]} \\
{[-19.9201,18.7750]}
\end{array}\right] .
\end{aligned}
$$


TABLE 2: Intermediate results of interval modified conjugate gradient method.

\begin{tabular}{lcc}
\hline$i$ & $x_{i}$ & $\max \left(\operatorname{Mag}\left(r_{i}\right)\right)$ \\
\hline 1 & {$[[-1.62482,1.62482],[-1.04453,1.04453],[-0.34818,0.34818]]^{\top}$} & 5.44647 \\
5 & {$[[-2.89491,2.89491],[-0.98549,0.98549],[-0.33668,0.33668]]^{\top}$} & 0.08503 \\
10 & {$[[-2.92550,2.92550],[-0.94522,0.94522],[-0.36736,0.36736]]^{\top}$} & 0.0037181 \\
20 & {$[[-2.92667,2.92667],[-0.94353,0.94353],[-0.36853,0.36853]]^{\top}$} & 0.0000036325 \\
26 & {$[[-2.92668,2.92668],[-0.94353,0.94353],[-0.36856,0.36856]]^{\top}$} & 0.000000056648 \\
\hline
\end{tabular}

TABLE 3: Intermediate results of interval steepest descent method.

\begin{tabular}{lcc}
\hline$i$ & $y_{i}$ & $\max \left(\operatorname{Mag}\left(r_{i}\right)\right)$ \\
\hline 1 & {$[[-3.25581,3.25581],[-0.95728,0.95728],[-0.36374,0.36374]]^{\top}$} & 1.43591 \\
3 & {$[[-2.92551,2.92551],[-0.94435,0.94435],[-0.36825,0.36825]]^{\top}$} & 0.00379605 \\
7 & {$[[-2.92667,2.92667],[-0.94353,0.94353],[-0.36853,0.36853]]^{\top}$} & 0.000013318 \\
11 & {$[[-2.92668,2.92668],[-0.94353,0.94353],[-0.36854,0.36854]]^{\top}$} & 0.000000046723 \\
\hline
\end{tabular}

It is to be noted that the solutions obtained from our methods are better than other techniques.
Example 8. Consider the linear interval system $\widetilde{A} \widetilde{x}=\widetilde{b}$, where $\widetilde{A}$ is a random diagonally dominant interval matrix

$$
\begin{gathered}
\widetilde{A}=\left[\begin{array}{ccccccc}
{[3.6178,6.4222]} & {[-0.0924,0.6944]} & {[-0.1140,0.7961]} & {[0.1062,0.5894]} & {[0.3416,0.4521]} & {[-0.4482,0.7578]} \\
{[0.3220,0.3508]} & {[4.0697,6.6729]} & {[-0.0517,0.2525]} & {[-0.1653,0.7593]} & {[-0.2746,0.5602]} & {[-0.0344,0.0681]} \\
{[-0.6805,0.7193]} & {[0.0603,0.6829]} & {[3.7386,6.7575]} & {[-0.1892,0.5012]} & {[-0.1225,0.2106]} & {[0.2003,0.4770]} \\
{[-0.5043,0.6541]} & {[0.0249,0.3366]} & {[-0.2728,0.8216]} & {[3.7887,6.9165]} & {[-0.1346,0.4485]} & {[0.3347,0.4588]} \\
{[-0.0464,0.5994]} & {[-0.2883,0.5930]} & {[0.1657,0.3306]} & {[-0.3346,0.7799]} & {[4.0636,6.4148]} & {[-0.0716,0.2109]} \\
{[0.1975,0.2994]} & {[-0.0125,0.2048]} & {[0.0754,0.1017]} & {[-0.2637,0.4392]} & {[-0.0188,0.0996]} & {[4.0029,6.4124]}
\end{array}\right], \\
\tilde{b}=\left[\begin{array}{c}
{[-5.6444,5.6444]} \\
{[-3.8203,3.8203]} \\
{[-1.7073,1.7073]} \\
{[-3.8776,3.8776]} \\
{[-4.6594,4.6594]} \\
{[-2.6347,2.6347]}
\end{array}\right]
\end{gathered}
$$

This system has exact solution

$$
\tilde{x}_{s}=\left[\begin{array}{c}
{[-0.7089,0.7089]} \\
{[-0.4365,0.4365]} \\
{[-0.0625,0.0625]} \\
{[-0.4072,0.4072]} \\
{[-0.5563,0.5563]} \\
{[-0.3263,0.3263]}
\end{array}\right] .
$$

We considered

$$
\begin{aligned}
& \tilde{x}_{0} \\
& =[[0,0],[0,0],[0,0],[0,0],[0,0],[-1,1]]^{\top},
\end{aligned}
$$

as initial solution and $\theta=10^{-3}$ as tolerance.
The generalized conjugate gradient method, after 7 iterations obtained, is

$$
\tilde{x}_{1}=\left[\begin{array}{c}
{[-0.70881,0.70881]} \\
{[-0.43651,0.43651]} \\
{[-0.06252,0.06252]} \\
{[-0.40720,0.40720]} \\
{[-0.55632,0.55632]} \\
{[-0.32634,0.32634]}
\end{array}\right] .
$$

while the value of magnitudes of residual $\left(\widetilde{A} \widetilde{x}_{1} \ominus b\right)$ is $5.94044 \times$ $10^{-5}$.

When $\theta=10^{-7}$, interval modified conjugate gradient method after 26 iterations obtained $\tilde{x}_{2}$ while magnitude of $\widetilde{A} \widetilde{x}_{2} \ominus b$ is $7.67731 \times 10^{-8}$. 


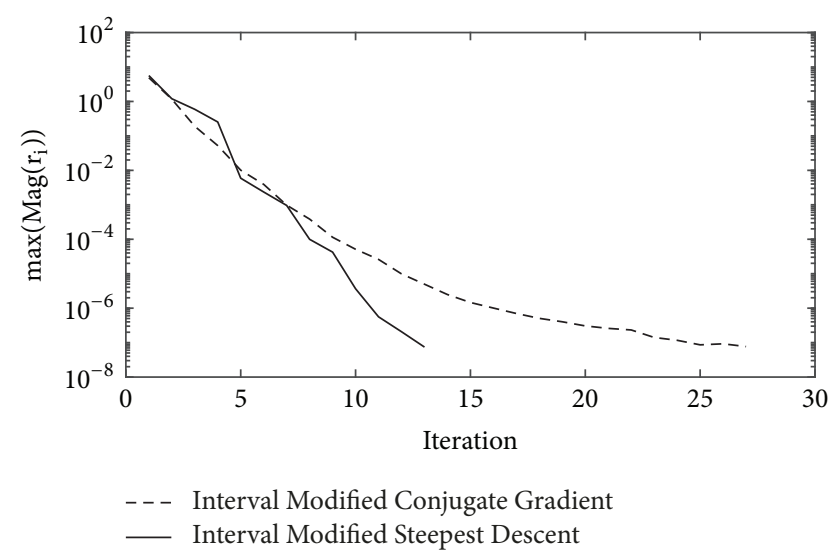

FIGURE 1: Tightness of solutions and convergences of our methods.

Interval steepest descent method obtained estimations $\tilde{y}_{1}$ and $\tilde{y}_{2}$ at iterations 6 and 12 when $\theta=10^{-3}$ and $\theta=10^{-7}$, respectively. The accuracy of $\tilde{y}_{1}$ and $\tilde{y}_{1}$ is

$$
\begin{aligned}
& \max \left(\operatorname{Mag}\left(\widetilde{b} \ominus \widetilde{A} \widetilde{y}_{1}\right)\right)=9.59448 \times 10^{-4}, \\
& \max \left(\operatorname{Mag}\left(\widetilde{b} \ominus \widetilde{A} \widetilde{y}_{2}\right)\right)=7.42146 \times 10^{-8}
\end{aligned}
$$

The results of these experiments are shown in Figure 1. This figure shows the convergence of the two proposed methods. As it can be seen, the interval steepest descent method has faster convergence than interval modified conjugate gradient method.

This example illustrates that our methods can solve systems where some of their elements have 0 in their intervals, without producing divide-by-zero error.

Example 9. Consider Example 8 with a different

$$
\widetilde{b}=\left[\begin{array}{c}
{[0,5.6444]} \\
{[0,3.8203]} \\
{[0,1.7073]} \\
{[0,3.8776]} \\
{[0,4.6594]} \\
{[0,2.6347]}
\end{array}\right],
$$

which has the exact solution

$$
\left[\begin{array}{l}
{[0,0.7089]} \\
{[0,0.4365]} \\
{[0,0.0625]} \\
{[0,0.4072]} \\
{[0,0.5563]} \\
{[0,0.3263]}
\end{array}\right] .
$$

Using interval modified conjugate gradient method, we obtain

$$
\widetilde{x}=\left[\begin{array}{l}
{[0.0000,0.70952]} \\
{[0.0000,0.43667]} \\
{[0.0000,0.05902]} \\
{[0.0000,0.40737]} \\
{[0.0000,0.55669]} \\
{[0.0000,0.32652]}
\end{array}\right] .
$$

Using interval steepest descent method, we obtain

$$
\widetilde{y}=\left[\begin{array}{l}
{[0,0.70898]} \\
{[0,0.43650]} \\
{[0,0.06248]} \\
{[0,0.40719]} \\
{[0,0.55629]} \\
{[0,0.32629]}
\end{array}\right] .
$$

Let the system considered in Example 8 be given with another $\widetilde{b}$

$$
\widetilde{b}=\left[\begin{array}{c}
{[1.2542,5.6443]} \\
{[1.6069,3.8202]} \\
{[0.4887,1.7072]} \\
{[0.9962,3.8775]} \\
{[1.4827,4.6593]} \\
{[1.1532,2.6346]}
\end{array}\right]
$$

which has the exact solution

$$
\left[\begin{array}{c}
{[0.2692,0.7089]} \\
{[0.3419,0.4365]} \\
{[0.0425,0.0625]} \\
{[0.1877,0.4072]} \\
{[0.3158,0.5563]} \\
{[0.2591,0.3263]}
\end{array}\right] .
$$

Using interval modified conjugate gradient method and interval steepest descent method, we obtain

$$
\tilde{x}=\left[\begin{array}{c}
{[0.26950,0.70873]} \\
{[0.34162,0.43654]} \\
{[0.04248,0.06475]} \\
{[0.18786,0.40723]} \\
{[0.31583,0.55638]} \\
{[0.25722,0.32627]}
\end{array}\right]
$$


TABLE 4: Iterations needed in interval steepest descend method solving random linear interval systems.

\begin{tabular}{lcc}
\hline$n$ & $\theta=10^{-3}$ & $\theta=10^{-7}$ \\
\hline 5 & 10 & 18 \\
10 & 13 & 33 \\
15 & 6 & 19 \\
20 & 16 & 36 \\
25 & 14 & 35 \\
30 & 18 & 40 \\
35 & 14 & 32 \\
40 & 15 & 36 \\
45 & 14 & 34 \\
50 & 18 & 40 \\
75 & 16 & 35 \\
100 & 18 & 44 \\
\hline
\end{tabular}

TABLE 5: Iterations needed in interval modified conjugate gradient method solving random linear interval systems.

\begin{tabular}{lcc}
\hline$n$ & $\theta=10^{-3}$ & $\theta=10^{-7}$ \\
\hline 5 & 8 & 23 \\
10 & 21 & 37 \\
15 & 24 & 52 \\
20 & 27 & 63 \\
25 & 34 & 87 \\
30 & 52 & 120 \\
35 & 80 & 186 \\
40 & 91 & 240 \\
45 & 67 & 247 \\
50 & 84 & 289 \\
75 & 167 & 412 \\
100 & 183 & 542 \\
\hline
\end{tabular}

and

$$
\tilde{y}=\left[\begin{array}{c}
{[0.26976,0.70892]} \\
{[0.34186,0.43651]} \\
{[0.04240,0.06251]} \\
{[0.18768,0.40720]} \\
{[0.31580,0.55630]} \\
{[0.25905,0.32630]}
\end{array}\right] .
$$

Example 10. We examined the two proposed methods with a number of random linear interval systems $\widetilde{A} \widetilde{x}=\widetilde{b}$ when $\widetilde{A} \epsilon$ $\square \mathbb{R}^{n \times n}$ is a random interval symmetric diagonally dominant matrix. Tables 4 and 5 show the number of iterations needed by our presented methods to find the solution with tolerances $\theta=10^{-3}$ and $\theta=10^{-7}$ for random interval systems with various dimensions.

Note that there are infinite random matrices with dimension $n \times n$, and number of iterations just does not depend on $n$. It depends on $n$, the coefficient matrix, and right-hand side vector. However, each row of these tables just shows number of iterations for random linear systems. These matrices are not special matrices, and these tables show a good view of dependency of dimension and approximation of number of iterations. These tables also show needed iterations in interval steepest descent are less than the interval modified conjugate gradient method.

\section{Conclusion}

In this paper, we presented two operators " $\ominus$ " and “ $\oslash$ " as inverse operators of " + " and " $x$ " in interval arithmetic. The proposed operation " $\oslash$ " can solve many equations in the form $\widetilde{a} \widetilde{x}=\widetilde{b}$, where $0 \in \widetilde{b}$, without concerning divide-by-zero.

Using these two operators, we proposed two iterative methods for computing exact solution of linear interval systems. The first method, based on the conjugate gradient method, replaced real operations with interval operations. The second method uses steepest descent idea to solve linear interval systems. We presented the interval function $\Phi(\widetilde{x})$. Then using analysis of $\Phi(\tilde{x})$, it was proved that the second method is convergent.

Our proposed methods are using operations " $\ominus$ " and “ $\oslash$," solving systems, specially systems which have 0 belonging to some of their elements without the divide-by-zero issues. This is the main advantage of our methods. Also, our results showed the efficiency and fast convergence of the proposed 
methods. In addition, one can control and improve the accuracy of the solution, by control of the tolerance parameter $\theta$.

The important application of interval number and interval analysis is in the modeling of uncertain values. There are many uncertain values in fields of engineering sciences that can be modeled in interval numbers. Uncertainty in some of these problems can be modeled in interval numbers, for example, the interval methods applied to linear state feedback control of uncertain values [41], reliability optimization under parameter uncertainty [42], decision-making under uncertainty [43], and perturbation analysis [44]. Our next research is to consider the applicability of our method to some of the engineering problems.

\section{Data Availability}

No data were used to support this study.

\section{Conflicts of Interest}

The authors declare that there are no conflicts of interest regarding the publication of this paper.

\section{References}

[1] G. H. Golub and C. F. van Loan, Matrix Computations, The Johns Hopkins University Press, Baltimore, Md, USA, 3rd edition, 1996.

[2] B. N. Datta, Numerical Linear Algebra and Applications, SIAM, Philadelphia, Pa, USA, 2010.

[3] M. J. Wierman, An introduction to the mathematics of uncertainty, Creighton University, 2010.

[4] Z. Pawlak, L. Polkowski, and A. Skowron, Rough Set Theory, 2008.

[5] H.-J. Zimmermann, "Fuzzy set theory," Wiley Interdisciplinary Reviews: Computational Statistics, vol. 2, no. 3, pp. 317-332, 2010.

[6] B. Liu, "Uncertainty theory," in Uncertainty theory, pp. 205-234, Springer, Berlin, Germany, 2007.

[7] G. Alefeld and J. Herzberger, Introduction to Interval Computations, Academic Press, New York, NY, USA, 1983.

[8] F. Mora-Camino and C. A. Cosenza, "Fuzzy Dual Numbers," in Fuzzy Dual Numbers, pp. 11-16, Springer, 2018.

[9] E. Zieniuk, A. Kużelewski, and M. Kapturczak, "The influence of interval arithmetic on the shape of uncertainly defined domains modelled by closed curves," Computational \& Applied Mathematics, vol. 37, no. 2, pp. 1027-1046, 2018.

[10] D. Pal and G. S. Mahapatra, "Parametric functional representation of interval number with arithmetic operations," International Journal of Applied and Computational Mathematics, vol. 3, no. 2, pp. 459-469, 2017.

[11] R. E. Moore, Interval Analysis, Prentice-Hall, Englewood Cliffs, NJ, USA, 1966.

[12] E. Hansen, "On the solution of linear algebraic equations with interval coefficients," Linear Algebra and its Applications, vol. 2, pp. 153-165, 1969.

[13] A. Neumaier, Interval Methods for Systems of Equations, Cambridge, UK, Cambridge University Press, Cambridge, 1990.
[14] K. Ganesan and P. Veeramani, "On arithmetic operations of interval numbers," International Journal of Uncertainty, Fuzziness and Knowledge-Based Systems, vol. 13, no. 6, pp. 619-631, 2005.

[15] S. Abolmasoumi and M. Alavi, "A Method For Calculating Interval Linear System," Journal of Mathematics and Computer Science, vol. 08, no. 03, pp. 193-204, 2014.

[16] M. Liu, "Interval Analysis of Dynamic Response of Structures," in Proceedings of the 2015 International Conference on Electrical, Automation and Mechanical Engineering, K. Chan and J. Yeh, Eds., Phuket, Thailand, July 2015.

[17] S. H. Chen, H. D. Lian, and X. W. Yang, "Dynamic response analysis for structures with interval parameters," Structural Engineering and Mechanics, vol. 13, no. 3, pp. 299-312, 2002.

[18] S. Marques, M. Beer, A. Gomes, and A. Heriques, "Limit state imprecise interval analysis in geotechnical engineering," in Geotechnical Safety and Risk V, T. Schweckendiek, A. F. D. Pereboom, M. Th. van Staveren, and P. M. C. B. M. Cools, Eds., pp. 193-204, IOS Press, 2015.

[19] D. N. Mohsenizadeh, V. A. Oliveira, L. Keel, and S. P. Bhattacharyya, "Extremal results for algebraic linear interval systems," in Optimization and its applications in control and data sciences, B. Goldengorin, Ed., vol. 115, pp. 341-351, Springer, 2016.

[20] E. D. Popova, "Multiplication distributivity of proper and improper intervals," Reliable Computing. An International Journal Devoted to Reliable Mathematical Computations Based on Finite Representations and Guaranteed Accuracy, vol. 7, no. 2, pp. 129-140, 2001.

[21] R. E. Moore, R. B. Kearfott, and M. J. Cloud, Introduction to Interval Analysis, SIAM, Philadelphia, Pa, USA, 2009.

[22] P. Karunakar and S. Chakraverty, "Solving fully interval linear systems of equations using tolerable solution criteria," Soft Computing, vol. 22, no. 14, pp. 4811-4818, 2018.

[23] S. P. Shary, "A new technique in systems analysis under interval uncertainty and ambiguity," Reliable Computing. An International Journal Devoted to Reliable Mathematical Computations Based on Finite Representations and Guaranteed Accuracy, vol. 8, no. 5, pp. 321-418, 2002.

[24] M. Hladik, "New operator and method for solving real preconditioned interval linear equations," SIAM Journal on Numerical Analysis, vol. 52, no. 1, pp. 194-206, 2014.

[25] M. Hladık, "Weak and strong solvability of interval linear systems of equations and inequalities," Linear Algebra and its Applications, vol. 438, no. 11, pp. 4156-4165, 2013.

[26] T. Nirmala, D. Datta, H. S. Kushwaha, and K. Ganesan, "Inverse interval matrix: a new approach," Applied Mathematical Sciences, vol. 5, no. 13-16, pp. 607-624, 2011.

[27] J. Zhen and D. den Hertog, "Centered solutions for uncertain linear equations," Computational Management Science, vol. 14, no. 4, pp. 585-610, 2017.

[28] E. D. Popova, "Outer bounds for the parametric controllable solution set with linear shape," in Proceedings of the International Symposium on Scientific Computing, Computer Arithmetic, and Validated Numerics, pp. 138-147, Springer, 2015.

[29] T. Nirmala, D. Datta, H. S. Kushwaha, and K. Ganesan, "On the solution of system of interval linear equations," International Journal of Applied Engineering Research, vol. 10, no. 6, pp. 15777$15797,2015$.

[30] T. Nirmala and K. Ganesan, "Modified Crout's method for an LU decomposition of an interval matrix," Journal of Physics: Conference Series, vol. 1000, pp. 1-11, 2018. 
[31] J. Rohn, "Inverse interval matrix," SIAM Journal on Numerical Analysis, vol. 30, no. 3, pp. 864-870, 1993.

[32] S. Markov, "On directed interval arithmetic and its applications," J.UCS. The Journal of Universal Computer Science, vol. 1, no. 7, pp. 514-526, 1995.

[33] E. Kaucher, "Interval analysis in the extended interval space IR," in Fundamentals of Numerical Computation (ComputerOriented Numerical Analysis), G. Alefeld and R. Grigorieff, Eds., pp. 33-49, Springer, Vienna, Austria, 1980.

[34] E. Siahlooei and S. A. Shahzadeh Fazeli, "An Application of Interval Arithmetic for Solving Fully Fuzzy Linear Systems with Trapezoidal Fuzzy Numbers," Advances in Fuzzy Systems-Applications and Theory, vol. 2018, Article ID 2104343, 10 pages, 2018.

[35] S. P. Shary, "Algebraic approach to the interval linear static identification, tolerance, and control problems, or one more application of Kaucher arithmetic," Reliable Computing. Nadezhnye Vychisleniya, vol. 2, no. 1, pp. 3-33, 1996.

[36] M. R. Hestenes and E. Stiefel, "Methods of conjugate gradients for solving linear systems," Journal of Research of the National Bureau of Standards, vol. 49, pp. 409-436 (1953), 1952.

[37] G. Allaire and S. M. Kaber, Numerical Linear Algebra, Springer, New York, NY, USA, 2008.

[38] D. G. Luenberger and Y. Ye, Linear and Nonlinear Programming, vol. 228, Springer, Berlin, Germany, 1984.

[39] J. Shewchuk and J. R. Shewchuk, An introduction to the conjugate gradient method without the agonizing pain, Carnegie Mellon University, Pittsburgh, Penn, USA, 1994.

[40] S. Ning and R. B. Kearfott, "A comparison of some methods for solving linear interval equations," SIAM Journal on Numerical Analysis, vol. 34, no. 4, pp. 1289-1305, 1997.

[41] M. Soylemez and N. Munro, "Robust pole assignment in uncertain systems," in Proceedings of the IEEE ProceedingsControl Theory and Applications, vol. 144, pp. 217-224, 1997.

[42] G. Levitin, L. Xing, and Y. Dai, "Optimal completed work dependent loading of components in cold standby systems," International Journal of General Systems, vol. 44, no. 4, pp. 471484, 2015.

[43] A. Soroudi and T. Amraee, "Decision making under uncertainty in energy systems: state of the Art," Renewable \& Sustainable Energy Reviews, vol. 28, pp. 376-384, 2013.

[44] B. Xia, D. Yu, and J. Liu, "Interval and subinterval perturbation methods for a structural-acoustic system with interval parameters," Journal of Fluids and Structures, vol. 38, pp. 146-163, 2013. 


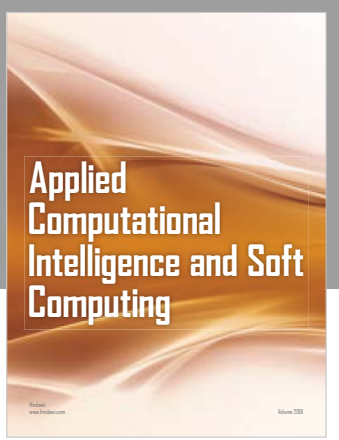

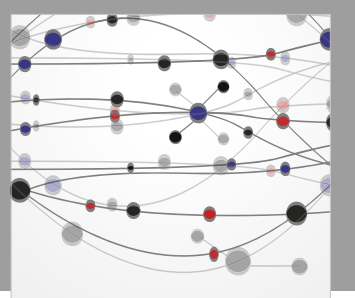

The Scientific World Journal
Submit your manuscripts at

Computing
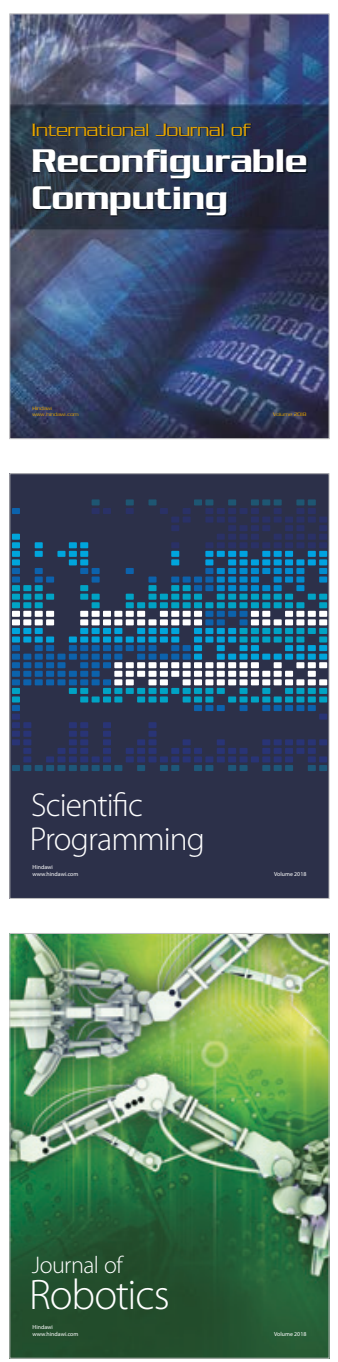

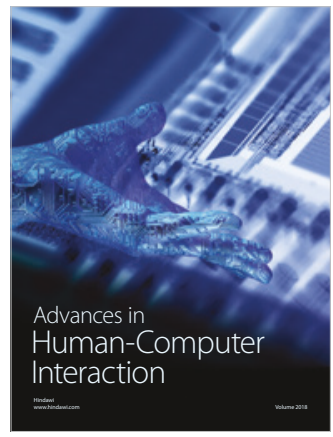

Human-Compute

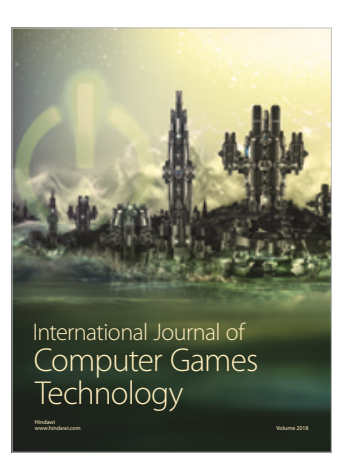

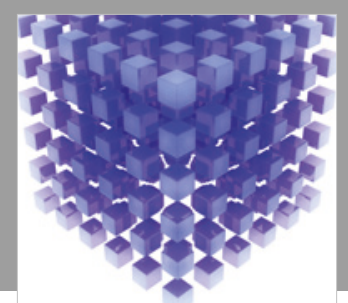

Mathematical Problems in Engineering

\section{Engincering}
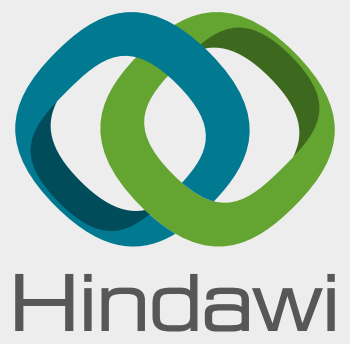

www.hindawi.com
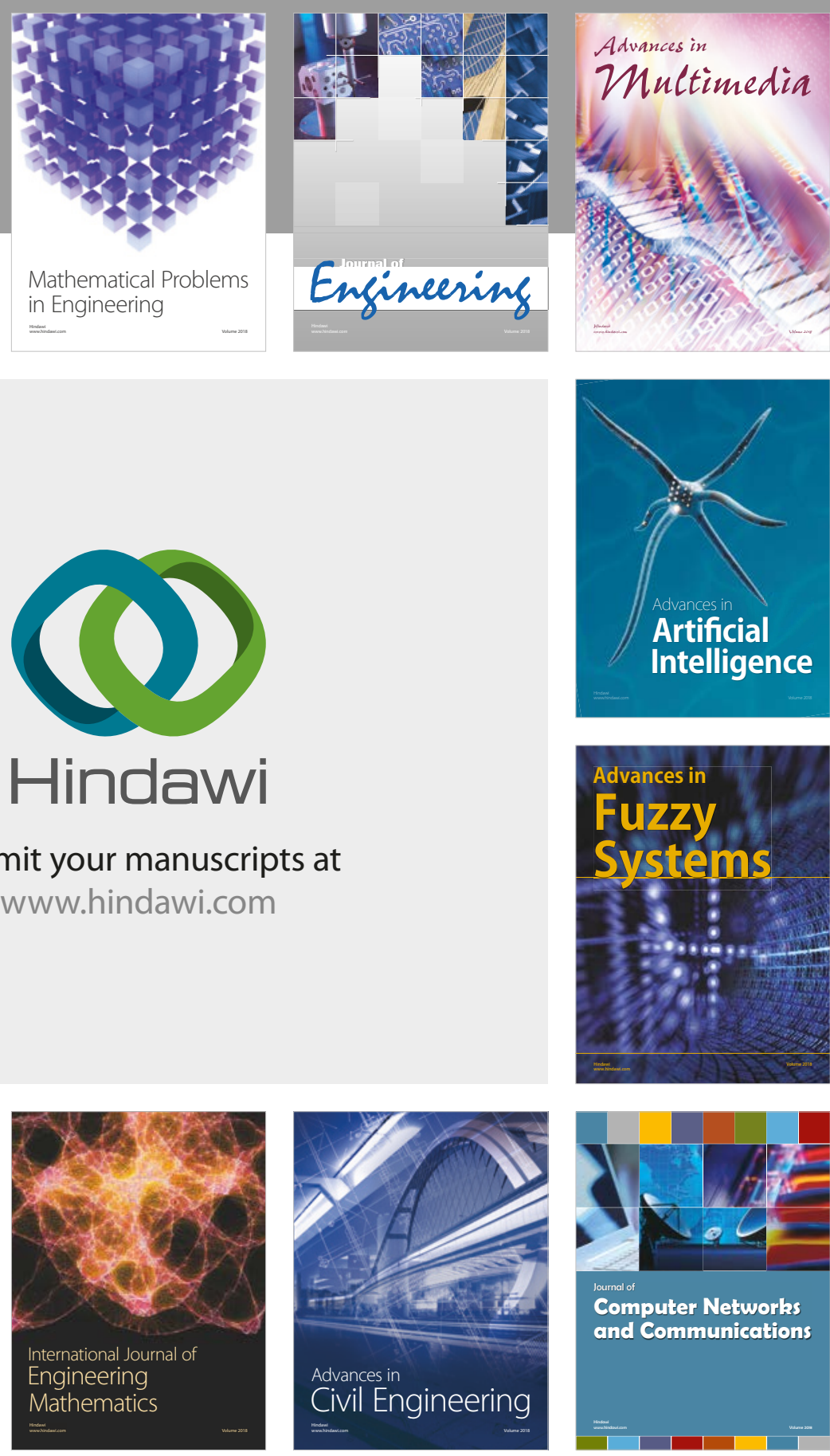

Computer Networks and Communications

Multimedia
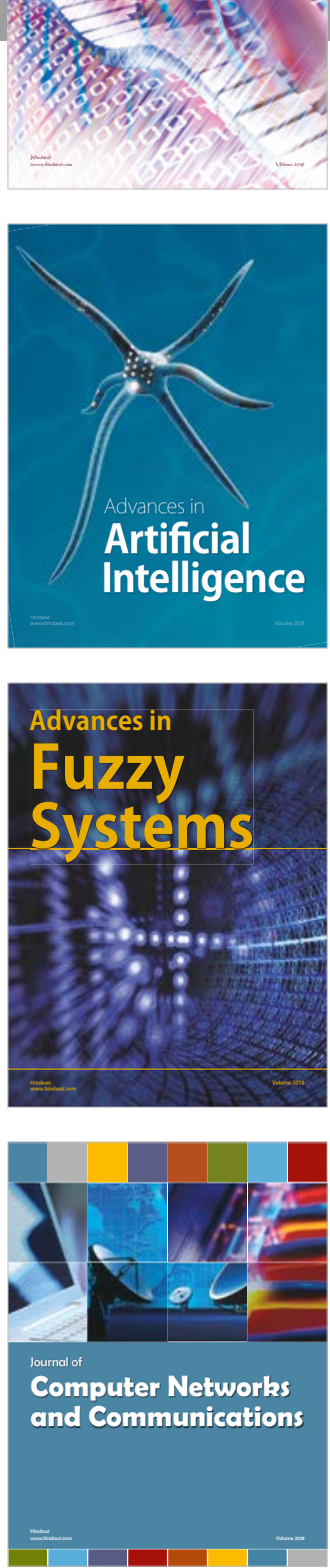

Advances in

Modelling \&

Simulation

in Engineering

interaction

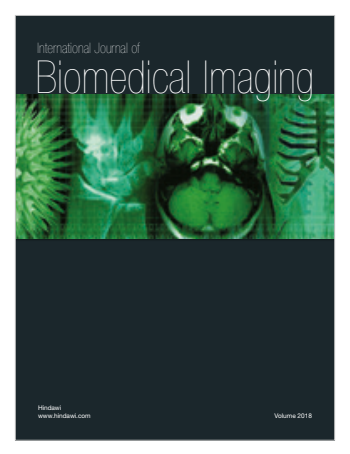

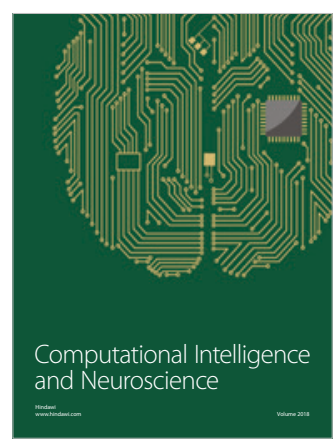

